\title{
W $\mathfrak{g} \mathfrak{r} \mathfrak{b} \mathfrak{e} \mathfrak{r} \mathfrak{i} \mathfrak{d}) \mathfrak{t}$
}

Dererfen $\mathfrak{A}$ asgabe vou 1771 .

Bei bem Snquiftionseridte, weldes ber YSfarrer, Der Barbier, bie $\mathfrak{B a}\{\mathfrak{e}$ und bie Şaubbälterin über bie

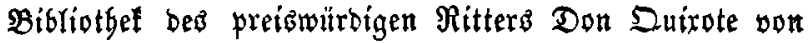
Manda im feqoiten Sapitel bes erften Theilo feiner Iebrreifen Gifidite Galten, find bie vier Bitwer bes

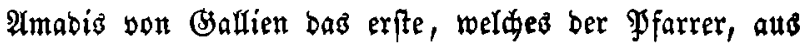

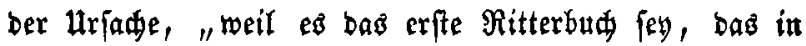
Spanien gebruat worben, tno weil es allen übrigen zum Mobell gebient babe," alo ben Stifter einer fo folimmen Secte, zum Feuer verbammt wiffen will. Der Barbier aber ftellt zum Bebuf beśfelben vor: "er babe von jefre berftänbigen Reuten fagen gebört, bafi es nidat nur bas erfte, fonbern aud bas befte unb eingige in feiner Art feb, weldeo die Spanier aufzutweifen bätten;" unb cr erbält burd feine färbitte, tá ibut ber 9 farrer wenig= fens bis auf weitere unterjuhung snabe wiberfabren.

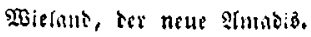


Iăpt. Das fïnfte Butd, weldes bie 2rbenteter bes Rai= fers Efplandian, bes älteften Solnes yon Amabis uno Driane, entgält, und bie folgenben adt Binder, worin bie Igaten ber Ritter Florifanbo, liowart, Ferion, Flos rifel doer âmabis aus Griedenlanto und feiner Söbne, Noger aus Griedentand tnb Siltio be $\mathfrak{l a}$ Silba, be= farieben finto, unb welde nad uno nad von verfibiebe= nen anbern, zum Theil unbefannten Berfaffern bingugetban worben, fitten als ttnedte Radahmungen eines Driginals, beffen eígener $\mathfrak{B}$ erth bem wadern $\mathfrak{y}$ farrer fdon megr

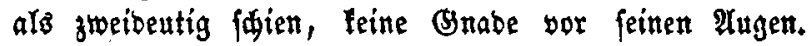
"Sie follen alle zum renfter Ginaus (jagt er, inbem er bie gamze zamilie bes gallifident 2 mabis bem meltlident

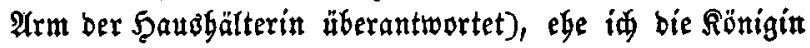

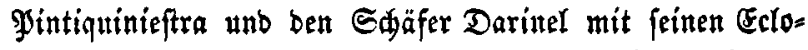
gen unt bie verwünfiften Disfertationen, bie ber $\mathfrak{A}$ utor affentbalben einmengt, werfionen wollte, ebe woulte id meinen Yeibliden $\mathfrak{B a t e r}$ fanmt ifnen verbrennen, wenn er mir in Beftalt eines irrenten $\Re$ titters in ben $\mathfrak{B u r f}$ fäme."

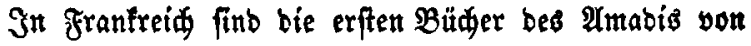
Riclas b' Serberay, 5eerrn Des Effars, überiełt und, yom Sagre 1540 an, nac uno nad Gerausgegeben, megr= mals aufgelegt unb in ber Forge von unterfaiebliden Berfaffern bis auf viet uno zwangig Büфer erweitert worben. 


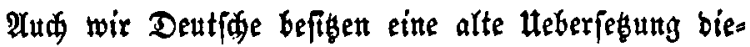
fes Ritterbuds, welde ben Spradforidern uno bemjenigen,

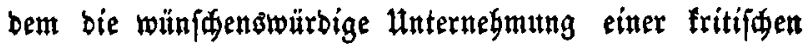
Geforidte ber beutfiden Sprade utto Riteratur vorbebals ten ift, nidt gleidgültíg feyn barf, and wowon aufer Der feltenten Folioausgabe von Gabre 1583, eine fpätere in vier und zwanzig biăen Drtavbänben, bie man niăt leidt beifanmen antrift, worganben iff.

Bernarbo $\mathfrak{x a f f}$, ber Bater bes Sängers Rinarbo's uno Bottfriebs, Gat biefem Stammuater fo vieler irren=

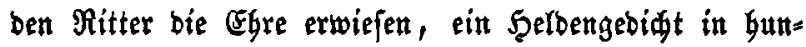
bert Gefängen uno melgr als fieben taufeno adtzeiligen Stanzen aus feiner Befdidte zut verfertigen; ein $\mathfrak{B}$ erf, beffen poetifie Berbienfte, nad einigen Stüfen von bem Ganzen zu urtbeilen, ziemlid weit hinter ben

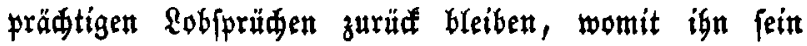
Borrebner, \&obovico Dolce, in zat yottem Make über= 低üttet bat.

Beber mit biefem Amadigi bes $\mathfrak{B}$ ernarbo $\mathfrak{x a}$ 角, nod) mit bem alten 2 trabis be (S)aule, now mit irgend einem anbern $\mathfrak{A m}_{\mathfrak{m} a b i s}$ in ber $\mathfrak{W e l t}$ gat ber gegenwärtige neue 2 mabis aufer bem Ramen (unt aufer berjentigen Iebnliafeit, bie er fogar mit ben Contes de ma mere

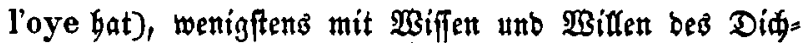
ters, nidjt bas Minbefte gemein. Die Raume, Deren 2Ats: geburt bas $\mathfrak{2 B e r f}$ felbit ift, bat igm aud ben $\mathfrak{R a m e n}$ 


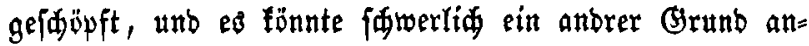
gegeben werben, warum diefes Ġebidt nidjt der nette Efplandian pber ber neue Floriônarte genannt worbent

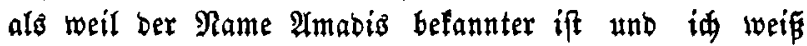

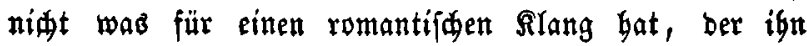
vorzüglid geifiaft madt, einen athenteurer son fo fon= Derbarem Sdjlage z̆u bezeidnen.

Die $\mathfrak{B}$ erşart, weldye unjer Didter zit einem $\mathfrak{B}$ erfe, worin bie Şelben alle, mebr ober weniger, närrifø, uno tie Feelbinnen, bis auf eine ober zwei, bie abgefdumad=

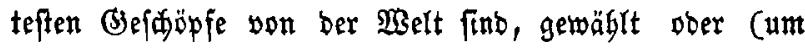
ism (Seredtigfeit wiserfabren zu Yaffen) erfunten hat,

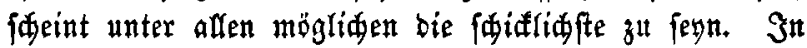

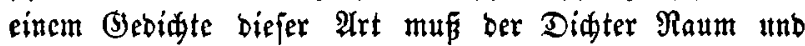
Freibeit genug baben, tamit ber (J)eift Capriccio, bem

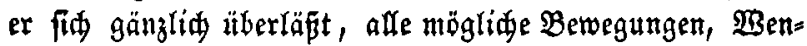
bungen uno Spränge maden fönne. Gebe einfürmige Bersart witrbe itgm einen Eang vorfdreiben, ber mit feinem Iaunigen Egarafter, mit ber $\mathfrak{M}$ unterfeit ano bem naiven Ton ber Erzäglung, mit bem Rä́lerliden ober Drolligen ber (S)egenftänbe, furz mit ber gattzen $\mathfrak{B e f}$ af $=$ fenteit eines Gebítes, weldes burdaus megr einem blopen Epielc ber \$rgantafie unb ber freimifligen Ergie= Fung einer reiden Brunnaber yon 2 Bis tho Sautne als

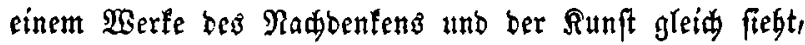
einen auffalfenben $\mathfrak{A}$ bfti maden wirbe. 
Die Bersant bes neuen $\mathfrak{T}$ ntabios Gat bie Bortheile Der meiften übrigen, obne ifre Mängel uno unbequem= lidfriten za baben. Sie jomiegt fich an alle arten von

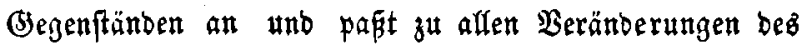
Tons unb Styls. Sie Gat, jentadoent es erforberlich ift, einen gelaffenen voer rafden, einen feierfidyen ober Güpfenben, einen eleganten voer nadläffigen Gang; fie windet fí, wie ein fanfter $\mathfrak{B a d}$, burd Blumentefitbe ober raufdt wie ein $\mathfrak{B a l t w a f f e r , ~ u ̈ b e r ~ S t a ̈ m m e ~ u n b ~ g e r f e n = ~}$

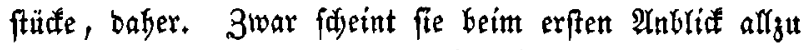
frei zu jeyn, um bem Poeten bie mintefte Mülle zu ver= arjadien: aber Hngelibte, bie ofne zartes Befübl für Rgytgmus und Sarmonie fie nadzuabmen verjudien woll= ten, mödten fid bierin betrogen finden. A Arfes in ber Worlt yat feine Regeln; unt bieje freie $\mathfrak{B e r b a r t ~ b a t ~ b e r e n t ~}$ viefleidit mely, als irgend cine andere. Sie if fäbig, wenn fie redift bebandelt wirb, einent (bebidite (boraus=

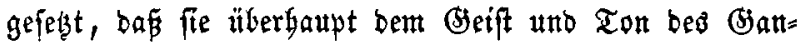

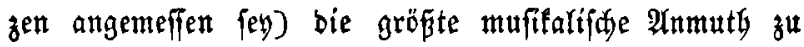
geben: aber unter angefdifften woer allzu naduläfigen 5̧änben würbe fie ein umerträglidjes Geleter werben. Die Nadabmer wiffen felten, wie viel Runult und weld eit

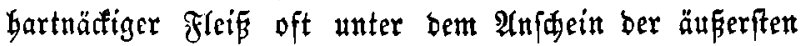
Reidtigfeit verftedt if. Tler forthe mant barum nidts Rettes wagen bürfen, bamit biefen unberufenten bie Beles genlyeit benwmmen mürbe, $\mathfrak{A}$ tôff 
Das Cigene biejer Bersant liegt, aufier ber Frcibeit, Berje von [ed) wedfeln za laffen, in ber bäufigen, ber $\mathfrak{B}$ Bilfür ober

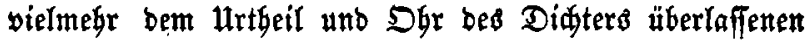

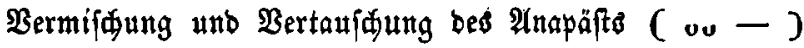
mit ben Samben, melde jonft bie berridente Berzart bes (sebidtes wären. Bielleidt wäre zat wüniden, baj bies

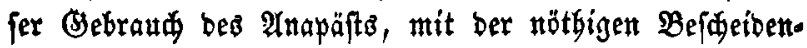
beit, aud in anbern Bebidyten, unb yornegmlid in werfificirten $\mathfrak{E}$ ft $=$ und Traueripielen, eingefülyrt würbe. Die Didter mürben baburd bes nadtheifigen unb nidit immer vermeibliden 3wanges entgoben, fid einer Menge

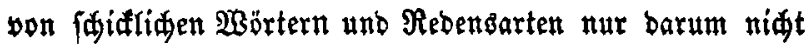
bebienten zu fönnen, weil fie nid)t in bie gewögnliden Jamben pafien; nidit zut gebenten, bap bie Monotonie, eine anbere, in langen Bebi iten jebr befwmerlide çigen= fdaft ber Iestern, baburi wenigftens gemiltert werben fönnte. Mande gute Gebiate mürben, burd biejes ein= zige Mittel, bon $\mathfrak{B o ̈ r t e r n , ~ b i e ~ n i d t ~ a n ~ i b r e m ~ S I l a g e ~}$ fethen, von Früllwörtern, Şärtigfeitert, ja jogar won Spradfeblern gereinigt merben, welde man bem 2 utor jestat, wiewobl ungern, zu gut halten muß, ba man bie

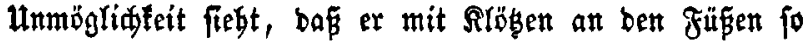
leiat und ungezwungen foutte tanzen fönnen, als of er

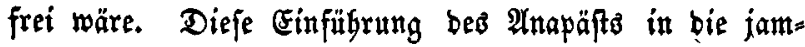
bifden Bersartcn wäre niđts weiter, als eine greibeit, 
beren fid fđon bie a्diten bebient Gaben, um igren $\Im a m=$ ben mebr Mannigfaltigfeit $\mathfrak{z}^{\mathfrak{u}}$ geben und fie in brama $=$

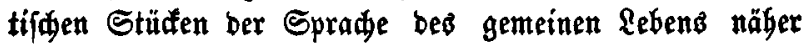
zu bringen. Hebrigens bedarf evi faum ber Grinnerung, Dafi bie $\mathfrak{B e r}[\mathrm{e}$ bes neuen $\mathfrak{A m a b i o s}$ (bis bas TaYent, Ge= bidte zu beclamiren, etwa einmal bei uns bie Beftalt einer förmliden §unft ergält,) mit gegöriger $\mathfrak{A}(\mathfrak{u}$ fmerlfam= feit ben Accent inmer babin zul fesen, wo ex bem Sinme ber Borte anb bem Ion ober affect bes Rebenben folge fieben mǘte, wenn fein beftimmtes Sylbenma vorganben wäre] wentigftend wie lebgafte $\mathfrak{3 r o f e}$ recitirt werben müffen; eine Regel, bie zwar getwiffermaken auf alle Bersarten anwenbbar ifa, aber bei biejer vor=

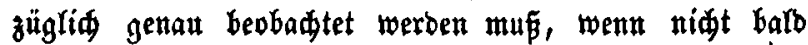

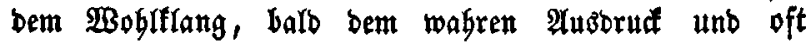
fogar bem Sinne ber Worte Serwalt gefwelyen foll.

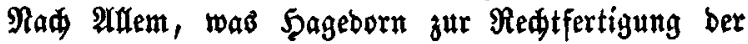
Îtmerfungen, womit er feine Srebidte mit beinabe ver= f gen einer Sdutgrede $\mathfrak{u m}$ fo weniger za bebürfen, ba man

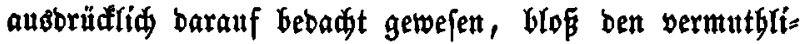

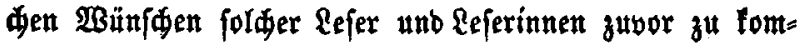

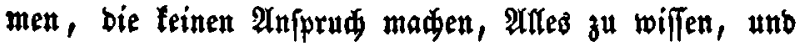
benen erlaubt ift, ofne Befdämung febre Bieles entweder nie gewulst pber wieber vergeffen zu baben; uno wern man fid babei nidyt immer auf bas blope Rotboürftige 
eingejdränft bat, fo gefdab es blos, weil man glaubte,

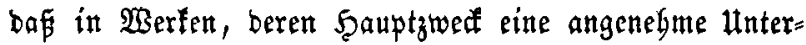

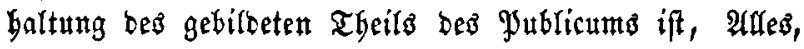
was biezat beitragen fann, obne ben $\mathfrak{E} e f e r$ gar zu weit aแจ bem İege zu fübren, an feinem redten Olağe ftebe. 